\title{
Finanzierungsmodelle und Anreizsysteme in der Suchtbehandlung aus deutscher und schweizerischer Perspektive
}

\author{
Ulrich Frick \\ Wulf Rössler
}

\author{
Modes of Reimbursement and Financial Incentives in Addiction Treatment in \\ Germany and Switzerland
}

\section{Zusammenfassung}

Die gesundheitspolitischen Zielsetzungen des ungehinderten Leistungszugangs, der Versorgungsgerechtigkeit, der Wirksamkeit und der Effizienz sind unmittelbar verbunden mit der Verfassung der Finanzierungswege in Versorgungssystemen. Die Mittelallokation von den Leistungseinkäufern zu den Leistungserbringern kann nach drei Systematiken mit jeweils unterschiedlichen Anreizwirkungen unterschieden werden: Variabilität versus Fixierung von Kosten, prospektive versus retrospektive Bezahlungsform und unterschiedlicher Pauschalisierungsgrad der Erstattungseinheiten. Für die Suchttherapien ist zudem ein unübersichtlicher Mix aus leistungsbezogenen Entgelten und institutioneller Förderung fast schon typisch und erschwert Evaluationen.

Die gegenwärtigen Reformen der Gesundheitssysteme bemühen sich zumeist um fixe Kosten, die prospektiv erstattet werden und einen zunehmend höheren Pauschalisierungsgrad für das entgoltene Paket von Dienstleistungen annehmen. Dies soll der Kostenbegrenzung und Effizienzsteigerung dienen. Methodisch suffiziente Evaluationen sind in überraschend geringer Zahl vorgelegt worden, so dass die Wirkungen der Finanzreformen erst nach und nach beschrieben werden können. Mit der Integration aller Finanzierungsformen der stationären Suchttherapien in ein einheitliches System wird in der Schweiz gegenwärtig ein radikaler und zukünftig beachtenswerter Systemwechsel vorbereitet.

\section{Schliuisselwörter}

Gesundheitssystem · Finanzierungsmodelle · Sucht · Effizienz

\section{Abstract}

Health policy goals of access, equity, effectiveness and efficiency are linked to the financing of the health care system. Three different categorisations of modes for allocating resources to the providers are usual: variable vs. fixed costs, prospective vs. retrospective payment and different levels of defining lump sums to be reimbursed. The field of substance abuse can additionally be characterised by a mixture of performance-based fees and institutional subventions which makes evaluation of financial incentives almost impossible.

Current changes in the health care system mostly strive for fixed costs, prospective payment and high levels of defining services. The goal of such changes is to limit costs and increase efficiency. However, there have been surprisingly few evaluations with good methodology. For inpatient addiction therapies Switzerland has created a radical change of financing system which should be taken into consideration for future developments.

\section{Key words}

Health care system $\cdot$ modes of reimbursement $\cdot$ substance abuse . efficiency 
Die 90er-Jahre waren eine Dekade von starken Reformbemühungen in den Gesundheitssystemen Westeuropas und einer völligen Revolutionierung der Gesundheitsversorgung in Osteuropa. In solch bewegten Zeiten ist es sinnvoll, sich als Ausgangspunkt für eine Darstellung von Finanzierungsmodellen den politischen Zweck zu nehmen, für den Versorgungssysteme organisiert werden. Die Weltgesundheitsorganisation hat dazu unlängst (Mai 2002) noch einmal ihre vier politischen Forderungen herausgestellt, denen sich die Ausgestaltung der nationalen Gesundheitsversorgung als Optimierungskriterien unterzuordnen hat:

1. „Access“ - Zugang zu Gesundheitsleistungen: Die Inanspruchnahme von Gesundheitsleistungen soll vom Bedarf und nicht von der Zahlungsfähigkeit, von räumlichen Distanzen oder von sozialen Stigmata gesteuert werden. Dass dies für die Suchtmedizin auch in entwickelten Marktwirtschaften noch ein einzulösendes Programm und nicht bestehendes Faktum ist, darauf weisen auch jüngst erschienene Publikationen erneut hin [1]. 2. „Equity“ als politisches Ziel wird im Deutschen meist mit „Versorgungsgerechtigkeit“ übersetzt. Aber gerade in der Suchtmedizin ist es nützlich, sich an die ursprüngliche Bedeutung dieser Forderung zu erinnern: Gleichheit bei der Verteilung von Ressourcen. Vor dieser Forderung nicht rechtfertigbare unterschiedliche Versorgungsgrade nach ethnischen Gesichtspunkten sind aktuell z.B. für i.v. Drogenkonsumenten [2] berichtet worden. 3. „Effectiveness“: Die Forderung, dass Leistungen des Versorgungssystems den Betroffenen nicht schaden dürfen und die Gesundheit tatsächlich verbessern sollen, ist auch im Bereich Mental Health durchaus keine redundante Selbstverständlichkeit, die sowieso schon als erfüllt gelten kann. Iatrogene Abhängigkeitserkrankungen (Schlafmittel, andere psychotrope Medikamente) zeugen davon, wie im Gesundheitssystem auch „negative Gesundheit“ produziert werden kann (vgl. schon 1978 [3]). Sogar gut gemeinte Präventionskampagnen (vgl. [4]) sind nicht davor gefeit, mehr Schaden als Nutzen zu erzeugen. 4. „Efficiency“: Mit der Gesamtmenge an Ressourcen, welche eine Gesellschaft für den Bereich „Mental Health“ bereitstellt, soll möglichst effizient umgegangen werden. Dazu müssen Vergleichsmaßstäbe vorhanden sein, wie über verschiedene Diagnosen und Therapieziele hinweg der Nutzen verschiedener Interventionen und ihr Ressourcenverbrauch gegeneinander abgewogen werden können (vgl. [5]). Diese vier Maximen sollen auch die Gedanken dieser Arbeit zu Finanzierungssystemen und Anreizstrukturen in der Suchttherapie führen.

\section{Geldfluss im Gesundheitssektor}

Im Gegensatz zu den üblichen Marktbedingungen, innerhalb derer der Konsument und der Leistungsanbieter direkt miteinander Geld und Dienstleistungen austauschen, laufen die Transfers im Gesundheitssektor wie in der Suchthilfe zum größten Anteil in einem Dreieck aus Bürgern, Leistungseinkäufern und Leistungserbringern (vgl. [6]).

So genannte „Out-of-Pocket“-Finanzierungen, bei denen der Gesundheitskonsument direkt aus eigener Tasche Leistungen kauft, sind in Deutschland im Jahr 1995 nur für 10,8\% der Gesundheits-

\begin{tabular}{|c|c|c|}
\hline & $\begin{array}{l}\text { Leistungstransfer } \\
\text { - Indikationsqualität } \\
\text { - Ergebnisqualität }\end{array}$ & \multirow[b]{2}{*}{ Leistungserbringer } \\
\hline Bürger & & \\
\hline Finanztransfer & & Mittelallokation \\
\hline $\begin{array}{l}\text { - Bedarfsgerechtigkeit } \\
\text { - Beitragsgerechtigkeit }\end{array}$ & $\begin{array}{l}\text { - Geld sammeln } \\
\text { - Risiko ausgleichen } \\
\text { - Leistung einkaufen }\end{array}$ & $\begin{array}{l}\text { - Preisbildung } \\
\text { - Kosteneffektivität } \\
\text { - Anreizstrukturen }\end{array}$ \\
\hline
\end{tabular}

Abb. 1 Trianguläre Finanzierungssituation im Gesundheitswesen.

ausgaben [7] verantwortlich gewesen, in der Schweiz für 28\% der Zahlungen im Jahr 1997 [8]. Das Geld der potenziellen Patienten fließt im Löwenanteil via Steuern oder Versicherungen schon vor jeder Inanspruchnahme zunächst zu den „Leistungseinkäufern“, die dann ihrerseits in einem oft aufwändig durchorganisierten Allokationsmechanismus die Leistungserbringer bezahlen, sobald diese an PatientInnen Leistungen erbringen. Sinn dieser Übung ist es, durch einen Risikoausgleich für die PatientInnen so genannte „katastrophale Risiken“1 aufzufangen. Damit soll der Zugang auch zu teuren Therapien für alle Teilnehmer gewährleistet werden (access!). Wir konzentrieren uns im Folgenden auf die Wege des Geldes, also auf die Pfeile von „Bürger“ zu „Leistungseinkäufer“ und von „Leistungseinkäufer“ zu „Leistungserbringer“ in Abb.1. Die Untersuchung der Leistungstransfers (mit welcher Indikations- und Leistungsqualität wird wem welche Gesundheitsleistung übermittelt?) würde den Rahmen dieser Darstellung sprengen.

\section{Wie wird (ein-)bezahlt?}

Neben den Bürgern eines Staates, die als Individuen, als Haushalte oder als Arbeitnehmer ihre Gesundheitsbeiträge in Form von Steuern, Pflichtversicherungsbeiträgen, freiwilligen Versicherungen, Rücklagenbildung und direkten Zahlungen leisten, treten meist auch Arbeitgeber als Finanzgeber im Gesundheitswesen auf. Deren Zahlungen erfolgen via Besteuerung und über Versicherungsbeiträge, aber nicht als „Out-of-Pocket“-Transfers. Der Staat tritt entgegen der ersten Erwartung hier keinesfalls als Financier des Gesundheitswesens zutage, sondern im Gegensatz dazu als Geldbezieher. Man muss sich vergegenwärtigen, dass es hier um den Prozess des Geldeinsammelns und noch nicht um den Prozess der Mittelallokation geht. Manche Staaten setzen mehr auf direkte und/oder indirekte Besteuerung, um ein Gesundheitssystem zu finanzieren, wie z.B. die skandinavischen Länder Dänemark, Finnland und Schweden [9]. Andere Länder orientieren sich eher am Bismarck'schen System einer Pflichtversicherung (z.B. Deutschland, Frankreich, die Niederlande).

Zunehmende Bedeutung erlangen in jüngerer Zeit freiwillige Versicherungen, mit denen vor allem diejenigen zusätzlichen Leistungen eingekauft werden können, die über die gesetzlichen Finanzierungswege ausgeschlossen werden. Zur Gänze aller-

\footnotetext{
${ }^{1}$ Ein „katastrophales Risiko“ ist für Ökonomen der finanzielle Ruin infolge teurer Krankheiten.
} 
dings wurde ein Gesundheitssystem selten auf freiwillige Krankenversicherungen basiert. Selten wurde ein Gesundheitssystem vollständig auf freiwillige Krankenversicherungen basiert. Noch seltener ist der Versuch, ohne ein „risk pooling“ die Finanzierung über privat zurechenbare, persönliche Rücklagen zu regeln. In Singapur (einem Land mit sehr hohem Pro-Kopf-Einkommen) bildet eine verpflichtende private Rücklagenbildung den Kern des Finanzierungssystems [10].

Als Geldbezieher unterscheiden Mossialos und Dixon [6] staatliche Exekutivorgane (vor allem Finanzämter) von den gemeinnützigen Körperschaften (z. B. Sozialversicherungen) als die beiden bedeutendsten Gruppen. Private Versicherungen (dritte Gruppe) können entweder mit Gewinnerzielungsabsicht oder gemeinnützig agieren. Sie sind für die Schweiz ein essenzieller Teil des Finanzierungssystems. Im Falle von direkten Zahlungen der PatientInnen an die Leistungserbringer treten Letztere neben ihrer Funktion als „Gesundheitsproduzenten“ dann zusätzlich als „Geldeinsammler“ (collection agent) auf.

Die Schweiz und Deutschland unterscheiden sich erheblich bei der Finanzierung der Gesundheitsausgaben. Während in Deutschland die Sozialversicherungen für gut zwei Drittel der Gesundheitsausgaben aufkommen, tragen gesetzliche Versicherungen in der Schweiz nur rund ein Drittel der Ausgaben [7, 8]. Zudem wird die obligatorische Krankenversicherung in der Schweiz von privaten Versicherungen auf For-Profit-Basis getragen und nur die Unfallversicherung („Invalidenversicherung“, wichtig für Reha-Maßnahmen) ist eine öffentliche Körperschaft.

Die gesundheitspolitischen Ziele des Zugangs (access) und der Fairness (equity) sind davon unmittelbar berührt, wenn sich unterschiedliche Bevölkerungsschichten eines Landes Gesundheitsleistungen in unterschiedlichem Ausmaß leisten können. Deutschland wird im World Health Report 2000 der WHO auf den weltweit sechstbesten Rangplatz hinsichtlich „finanzieller Fairness" gereiht. Der zugrunde liegende Fairness-Index (vgl. [11]) erfasst vor allem, wie stark der Anteil, den die Haushalte von ihrem disponiblen Einkommen für eine hinreichende Deckung „katastrophaler Risiken“ aufwenden müssen, über die verschiedenen Bevölkerungsschichten hinweg streut. Die Schweiz landet in dieser Beurteilung abgeschlagen auf Platz 38 aller untersuchten Staaten. Während in Deutschland die Krankenpflichtversicherung als prozentualer Beitragssatz vom Arbeitseinkommen abgezogen wird, Reiche und Arme also proportional gleich viel bezahlen ${ }^{2}$, wirkt sich die Schweizer Finanzierung über risikoadjustierte, fixe Kopfprämien ${ }^{3}$ regressiv aus, d.h., einkommensstarke Haushalte leisten einen relativ geringeren Gesundheitsbeitrag. Auch Out-of-Pocket-Zahlungen wirken „regressiv“. Einzig der Steueranteil an der Finanzierung des Gesundheitswesens kann in der Schweiz als (im ökonomischen Sinne) „pro-

\footnotetext{
${ }^{2}$ Dies gilt für den Großteil der Bevölkerung. Es besteht jedoch die Einschränkung, dass jenseits einer Beitragsbemessungsgrenze Spitzenverdiener aus der Pflichtversicherung aussteigen können und Beamte wie Selbständige nicht Teil der Pflichtversicherung sind.

${ }^{3}$ Die Prämien werden nach Alter und Geschlecht gestaffelt. Zudem kann der Versicherte unterschiedlich hohe Selbstbehalte („Franchise“) wählen, um seine Prämie zusätzlich abzusenken. Anders als in Deutschland sind in der Schweiz auch HMO-Versorgungsmodelle und GatekeeperVersicherungsmodelle als Pflichtversicherung vereinbar (vgl. [12]).
}

gressiv" bezeichnet werden, weil die Besteuerung der Einkommen (leicht) mit ihrer Höhe ansteigt. Bedenkt man, dass die Risiken für psychiatrische Erkrankungen nicht von der Schicht unabhängig sind, so stellt sich das Schweizer Finanzierungssystem besonders für PsychiatriepatientInnen nicht gerade als hoch solidarisch verfasst heraus (vgl. auch [9]).

\section{Wie wird (aus-)bezahltt?}

Der Prozess der Mittelallokation (vgl. Abb.1) im medizinischen Sektor berührt ganz unmittelbar die gesundheitspolitischen Grundforderungen nach Zugang („access“), Fairness („equity“) und Effizienz. Wenn für eine bestimmte Leistung nur die Kosten unterdeckenden Summen an die Leistungserbringer ausbezahlt werden, dann führt dies dazu, dass die entsprechende Leistung immer seltener angeboten und damit der Zugang erschwert wird. Wird umgekehrt eine Leistung (z. B. apparative Diagnostik oder High-Tech-Medizin) attraktiv entlohnt, dann entsteht ein Sog, diese Leistung auch über das gesundheitlich Notwendige hinaus „an den Mann/die Frau“ zu bringen [13]. Das Prinzip des „nihil nocere“ kann dadurch gefährdet werden. Dass keine Finanzierungsform im Gesundheitswesen ohne Fußangeln und Fallstricke ausgestattet ist, formuliert Berkeley-Professor James Robinson so: „There are many mechanisms for paying physicians; some are good and some are bad. The three worst are fee-for-service, capitation, and salary“ [14].

Entgegen der Erwartung, dass immer dann, wenn es ums Geld geht, ein Sachverhalt besonders gründlich evaluiert werde, erweist sich die mit empirischen Daten argumentierende Literatur zum Thema „Finanzielle Anreizsysteme im Gesundheitswesen“ als ziemlich spärlich. Drei unlängst erschienene systematische Reviews $[13,15,16]$ identifizieren in der Flut von über 5300 Veröffentlichungen zum Thema „Anreizsysteme“ nur acht randomisierte Studien, zwei Beobachtungsstudien mit angemessenem Kontrollgruppenansatz sowie eine Studie mit einem zeitreihenanalytischen Ansatz. Auch diese Studien sind aber methodisch durchaus nicht unproblematisch (vgl. [15]). Für die unklare und heterogene Ergebnislage in den Reviews sind nach unserer Einschätzung zwei Probleme wesentlich:

1. Durchgängig zu wenig beachtet wird das „Level-of-Unit“-Problem: Auf welcher Ebene wird interveniert und auf welcher Ebene wird gemessen? Neue Versicherungsmodelle für PatientInnen können nicht nahtlos an Daten von Arztpraxen evaluiert werden, neue Abrechnungsmodelle für Ärzte nicht nahtlos am individuellen PatientInnenverhalten (vgl. [17] für eine Simulation sich ergebender Fehlschlüsse und [18] für eine angemessene Analyse von Multilevel-Daten). 2. Die untersuchten finanziellen Anreize waren zumeist im Zuge von politischen Reformen ausgehandelte, ad hoc entworfene Maßnahmen(-bündel) und keineswegs systematisch aufeinander aufbauende Bausteine. Eine systematische Einordnung ökonomischer Anreiz-Wirkungen setzte eine einheitliche Taxonomie voraus, wie sie von Jegers u. Mitarb. [19] erst vor kurzem vorgelegt wurde. Wir skizzieren im Folgenden die von ihnen vorgeschlagenen drei Betrachtungsebenen: 


\section{Variabilität: fixes versus steigerbares Einkommen}

„Variabel“ heißt eine Bezahlform, wenn jede zusätzliche Leistungseinheit den Geldbetrag erhöht, den der Therapeut erhält. „Fixiert“ nennen Jeger et al. auf der Mikro-Ebene (Ärzte, Krankenhäuser) Bezahlformen, bei denen diese Kopplung „Zusatzleistung führt zu Zusatzentgelt" aufgehoben ist. Auf der Makro-Ebene des gesamten Gesundheitssystems verstehen sie unter einer „fixierten“ Finanzierung die so genannten „gedeckelten“ Budgets. Variable Erstattungen lösen nach der ökonomischen Theorie eine Produktion von Gesundheitsgütern so lange aus, bis der Kostenzuwachs beim Herstellen zusätzlicher „Gesundheitseinheiten“ den damit erzielbaren Einkommenszuwachs überholen würde. Bei profitablen Preisen ist damit ein starker Anreiz zur Überproduktion gegeben. Bei fixen Erstattungen entsteht ökonomisch ein starker Anreiz zu Kostensenkungen. Dies kann über Rationalisierungen oder durch Reduktion von Teilleistungen erreicht werden, was die Gefahr von Qualitätsausdünnung und Unterversorgung heraufbeschwört. Daher sollte bei entsprechenden Finanzierungsformen simultan auch ein effizientes Qualitätsmonitoring vereinbart werden.

\section{Zeitperspektive: ex post versus ex ante}

In „prospektiven“ Finanzierungssystemen werden die zu erstattenden Beträge vor der Leistungserbringung „ex ante“ festgelegt. Dabei kann diese Festlegung variabel oder fix organisiert werden. In der umgekehrten Zeitperspektive, also bei „retrospektiver" Erstattung, wäre eine „fixe“ Festlegung der Summe im Sinne der Entkopplung von Zusatzleistung und Zusatzeinkommen unsinnig. Der Leistungsanbieter hätte zum Zeitpunkt der Leistungserbringung keinerlei Orientierung über seine Wirtschaftlichkeit. Findet bei prospektiver Leistungserstattung parallel ein wirksames Qualitätsmonitoring statt, dann stimuliert dieses Entlohnungssystem die Effizienz der Leistungserbringung. Das finanzielle Risiko wird bei einer variablen und retrospektiv angelegten Kostenerstattung voll vom Zahler übernommen. Im Falle eines variablen und prospektiv angelegten Finanzierungssystems teilen sich der Leistungserbringer und der Zahler das finanzielle Risiko. Ein fixiertes und prospektives Entgeltsystem schließlich überwälzt das Risiko voll auf den Leistungserbringer.

\section{Erstattungseinheit: en gros versus en detail}

Als „Erstattungseinheit“ bezeichnet man ein gemeinsam abrechnungsfähiges Bündel an ärztlichen und anderen Teilleistungen. Am detailliertesten sind Abrechnungen auf ,fee for service“-Basis (FFS). FFS zeichnet sich durch die gute Verfügbarkeit preislich profitabler Einzelleistungen (access!) aus. Aber: Die Indikationsstellung für die Durchführung von FFS-Leistungen erfolgt verstärkt bei den qualitativ hochwertigen (und somit meist hochpreisigen und hochprofitablen) Leistungen. Apparative technologische Leistungen sind in FFS-Systemen leichter quantifizierbar als intellektuelle Leistungen (z. B. ein Arztgespräch) und somit häufiger. Innovationen brauchen länger in die breite Praxis, weil sie zuerst in den Leistungskatalog erstattungsfähiger Maßnahmen formell aufgenommen werden müssen.

Tagesgleiche Pflegesätze bildeten im stationären Bereich über lange Zeit und vielerorts die Abrechnungsbasis. Weil die Tagessätze besonders in somatischen Kliniken das reale Leistungsgeschehen nicht abbilden (bei gesundenden Patienten verläuft es mit degressiver Intensität), sind die Pflegetage gegen Ende ei- nes stationären Aufenthalts besonders profitabel für das Krankenhaus: ein starker Anreiz zur Verweildauerverlängerung. Nun sind zwar die durchschnittlichen Verweildauern in den letzten 40 Jahren überall zumeist gesunken, trotz Entlohnung nach Tagessätzen. Aber - so entgegnet die ökonomische Theorie - mit einem pauschalierten Entgeltsystem hätte dieser vornehmlich technologisch bedingte Rückgang noch viel stärker ausfallen können.

„Fallpauschalen“ (prospektiv) sind das gegenwärtige Zauberwort in der Diskussion um die Krankenhausfinanzierung. Dabei wird als Abrechnungseinheit der gesamte für die Behandlung notwendige Krankenhausaufenthalt betrachtet. Abgrenzungsschwierigkeiten für Fallpauschalen sind daher immer verstärkt bei chronischen Krankheiten zu erwarten, bei denen einzelne, die Fallpauschale begründende Krankheitsepisoden schlechter aufzeigbar sind. Fallpauschalen inkludieren einen starken ökonomischen Anreiz zur Verweildauersenkung und fördern die technologische Effizienzsteigerung (für Österreich vgl. [20], für Portugal vgl. [21]). Dies sind die Vorteile. Die Nachteile sind ebenfalls weithin bekannt: Fallpauschalen sind anfällig für „Rosinenpickerei“ (cream skimming), wenn sich konsequent profitsuchende Anbieter nur die einträglichsten Fälle aussuchen. Oft kann auch eine Ausweitung von Doppeldiagnosen beobachtet werden oder eine epidemiologisch nicht erklärbare Intensivierung von Krankheitsbildern (Upcoding).

Das Zauberwort der Zukunft (in Amerika: der Gegenwart) bei den Entgeltsystemen heißt „capitation“ und meint Kopfpauschalen. In Österreich erhalten beispielsweise Hausärzte pro gesehenem Patienten für jedes Quartal eine Pauschale, die die meisten Leistungen dieses Quartals begleichen soll. In der Schweiz existieren im ambulanten Versorgungsbereich seit einigen Jahren bereits HMO-Praxen (Health Maintenance Organization) nach amerikanischem Vorbild [22], deren Inanspruchnahme von den Versicherungen mit den Betreibern ebenfalls pauschaliert verrechnet wird. Einige „perverse incentives“ von Fallpauschalen (z.B. Erhöhung der Wiederaufnahmerate, Upcoding) werden in einem solchen System der Kapitation umgangen. Es macht keinen Sinn mehr, den Krankheitsverlauf eines Patienten in möglichst viele Behandlungsepisoden zu zerlegen. Auch ist die Diagnosestellung sicherlich unbefangener möglich, wenn keine Kopplung zwischen erstellter Diagnose und Höhe der erzielbaren Vergütung besteht. Aber das Risiko des verhinderten Leistungszugangs ist den kopfpauschalierten Entgeltsystemen nach wie vor immanent [23]. In Österreich wurde ein Teil der im Europavergleich sehr hohen Krankenhaushäufigkeit in Verbindung gebracht mit dem pauschalierten Abrechnungssystem im ambulanten Sektor (vgl. [24]). Allerdings sind HMOs als Weiterentwicklung der Kapitation je nach Vertrag auch für die stationäre Versorgung mit zuständig ${ }^{4}$ und somit „immun“ gegen einen solchen „Abschiebedruck“.

\footnotetext{
${ }^{4}$ Neben stationären Leistungen werden auch fachpsychiatrische Leistungen von den Schweizer HMOs zumeist zugekauft. Mit einem Kostenanteil von $26,4 \%$ an den ambulanten externen Kosten für solche Zukäufe ist die psychosoziale Betreuung in den gegenwärtigen HMOs ein im Vergleich zu anderen Versicherungsmodellen überrepräsentiertes Versorgungserfordernis [25].
} 
Die Leistungseinkäufer drängen daher auf eine noch stärker pauschalierte Entgeltform. Chaix-Couturier et al. nennen ein solches System dann „capitation per physician“ im Gegensatz zur eben beschriebenen "capitation per patient“ [13]. Mit institutionsübergreifenden, auf eine definierte Population bezogenen Globalbudgets wird dem Patienten-Verschieben der ökonomische Hintergrund entzogen. Ein Entgeltsystem, das es einem psychiatrischen Krankenhaus ermöglichen würde, innerhalb eines jährlichen Regionalbudgets seine PatientInnen in einem beliebig aus kürzeren und längeren stationären oder teilstationären Aufenthalten kombinierbaren System zu behandeln, und das auch „nachgehende Versorgung“ im Sinne von ambulanten, auch außerhalb des Krankenhauses agierenden Behandlungsteams mit einschlösse, wäre hier einzureihen.

Je pauschalierter sich Abrechnungssysteme entwickeln, umso ähnlicher werden sie einer leistungsunabhängig ausbezahlten, institutionellen Förderung. Im Suchthilfebereich sind solche „inputorientierten “ Finanzierungsformen (im Gegensatz zu den bisher beschriebenen „outputorientierten“ Leistungsentgelten) keine Seltenheit [26, 27]. Eine gute Kenntnis des jeweils gültigen Zuwendungsrechtes mit seinen Spielarten von Voll- und Teilfinanzierung, Festbetrags- oder Fehlbedarfsfinanzierung (für eine genaue Analyse vgl. [26]) stellt in der Suchthilfe daher einen wichtigen Beitrag zum institutionellen Überleben dar. Die meisten Einrichtungen finanzieren sich über eine Mischform von institutioneller Förderung und auf Klienten bezogenen Leistungsentgelten (vgl. auch [28]).

\section{Erfahrungen mit prospektiv-pauschalen Entgeltsystemen}

Bei den Finanzierungsreformen im Krankenhaussektor wurden in Deutschland die Psychiatrien von der Abrechnung via DRGs ${ }^{5}$ ausgenommen. Deutschland bedient sich der Australian-Refined-(AR-)Version des DRG-Systems [29, 30] zur Abrechnung ab 2003. Auch bei den schweizerischen Bestrebungen, die mittlerweile in den Kantonen Zürich und Vaud konkret Gestalt angenommen haben [31], wird die Psychiatrie jeweils aus der Abrechnung via $\mathrm{AP}^{6}$-DRGs herausgehalten. Es ist aber zu erwarten, dass diese Sonderrolle der Psychiatrie Anlass für weitere Diskussionen sein wird. Nach den ungünstigen Erfahrungen mit dem DRGSystem [32] in der Psychiatrie in den 80er-Jahren in den USA [33] haben nur wenige Länder den erneuten Versuch unternommen, stationär-psychiatrische Behandlungen mittels Fallpauschalen zu finanzieren. Österreich allerdings hat im Rahmen seiner „Leistungsorientierten Krankenanstaltenfinanzierung“ (LKF, [34]) ein eigenes Fallpauschalensystem unter Einbeziehung der Psychiatrie entwickelt und es im Jahre 1997 landesweit eingeführt (vgl. [35] für eine Kurzbeschreibung des Systems). Bei der LKF handelt es sich in der Terminologie dieser Arbeit um ein Hybridsystem, das Fallpauschalen (= prospektive, fixierte Abrechnung) mit degressiv ausgestalteten Tagesentgelten (= retrospektiv, variabel) und FFS-Pauschalen (retrospektiv, variabel) kombiniert. Mittlerweile existieren verschiedene Arbeiten zur Evaluation dieses

\footnotetext{
${ }^{5}$ Diagnosis Related Groups

${ }^{6}$ AP steht für „All Patients“ - auch wenn der geplante Einsatz die psychiatrischen Patienten nicht inkludiert!
}

Entgeltsystems, aber vornehmlich mit dem Blick auf die Effizienz einzelner Krankenhäuser [36, 37, 20]. Eine Public-Health-Perspektive, die die Auswirkungen auf die Versorgung einer ganzen Bevölkerung evaluiert, wurde nur für das Bundesland Salzburg vorgelegt [35]. Speziell für die stationäre Psychiatrie ließ sich zeigen, dass die LKF die ungünstigen Auswirkungen früherer Versuche mit DRG-ähnlichen Systemen offenbar vermieden hat und keine zusätzliche Verweildauerverkürzung unter Beschleunigung der so genannten Drehtür-Psychiatrie angeregt wurde [38]. Offen ist jedoch, ob sich die LKF in irgendeiner Weise auf das Behandlungsergebnis ausgewirkt hat.

Für die Abrechnungssysteme mit Kapitation erscheinen nach und nach methodisch gute Evaluationsarbeiten: Im US-Bundesstaat Colorado wurden im Jahr 1995 vierzehn der siebzehn MedicaidVersorgungsregionen im Bereich Mental Health auf Kapitation (zwei verschiedene Varianten) umgestellt, drei Regionen verblieben unter FFS-Abrechung und dienten als Kontrollgruppe. Neu gegründete, gemeinnützige „Mental Health Assessment and Service Agencies“ (MHASA) versuchten in sechs Regionen, Kostensenkungen vor allem durch geringere Hospitalisierungszahlen zu erreichen („direct capitation“). In den acht „teuersten“ Regionen wurde die Versorgung an eine privatwirtschaftliche „Managed Behavioral Health Organization“ (MBHO) vergeben, die ein ausgeklügeltes System der Patientenbetreuung mit dichten Behandlungsketten und Nachbetreuung organisierte. Die Evaluation verfolgte $522 \mathrm{Zu}$ fallspatientInnen über zwei Jahre [39]. Die Kosten sanken um 20\% beim System der „direkten Kapitation“, um 66\% unter den MBHOBedingungen und bei FFS nur unwesentlich.

In den Gebieten mit „direct capitation“ wurde zwar eine klare Reduktion der stationären Inanspruchnahme erreicht: Nur 77\% der chronisch kranken PatientInnen aus dem Ausgangsjahr wurden im zweiten Jahr nach Finanzierungsumstellung erneut hospitalisiert. Aber die befragten PatientInnen verbanden dies mit dem (im Vergleich zur FFS-Referenzgruppe) verstärkten Gefühl, es seien Betreuungs- und Dienstleistungen verweigert worden und die Wartezeit für einen Behandlungstermin sei gegenüber FFS-Bedingungen verlängert. Anders die MBHO-Regionen: Obwohl auch hier die Hospitalisierungen zurückgingen (auf $68 \%$ des Ausgangsniveaus), berichten die PatientInnen von einer gesteigerten Inanspruchnahme psychiatrischer Dienste, neuartigen Dienstleistungen, häufigeren vorgeplanten Kontakten mit dem Behandlungssystem und kürzeren Wartezeiten am einbestellten Termin.

Es scheint so, dass durch ein dichter geknüpftes, kontinuierliches Betreuungsnetz im ambulanten Behandlungssektor in den MBHOs nicht nur radikale Kostensenkungen durchgesetzt werden konnten, sondern dass sich auch in der subjektiven Sicht der PatientInnen eher eine Verbesserung der Versorgungssituation ergeben hat. Was die Ergebnisqualität anbelangt, konnten in einer detaillierten Analyse des Behandlungsoutcomes keine systematischen Unterschiede zwischen den Finanzierungsformen gefunden werden [40].

Ähnliche Ergebnisse berichten Magura et al. [41] über die Auswirkungen der im Staat Massachusetts 1992 vergebenen staatsfinanzierten Betreuung von psychiatrischen PatientInnen (Mental Health und Sucht) an eine private For-Profit-Organisation (MBHO-Prinzip wie in Colorado). In den beiden auf die Einfüh- 
rung von Managed Care folgenden Jahren sanken in Massachusetts im Bereich der Suchthilfe die Ausgaben um 48\%. Nach vier Jahren lagen sie immer noch um $42 \%$ unter dem Ausgangsniveau. Die Kostensenkung wurde nicht durch einen erschwerten Zugang zum Hilfesystem erkauft. Es zeigte sich vielmehr, dass die Rate der Inanspruchnahmen kontinuierlich stieg (um $43 \%$ ), und zwar besonders deutlich bei der Methadon-Substitution und den tagesklinischen Angeboten. Auch die Verweildauer stationärer Aufenthalte sank nicht, sondern verlängerte sich um $2 \%$. Die dramatische Kostensenkung konnte durch eine Verlagerung bislang vorwiegend im teuren vollstationären Bereich erbrachter Entgiftungen in den teilstationären und ambulanten Bereichen erzielt werden.

Ist der gesundheitsökonomische Stein der Weisen damit gefunden? Es muss bedacht werden, dass die Studien jeweils in der Versorgung von Medicaid-, Medicare- oder Veterans-Administration-PatientInnen unternommen wurden. Deshalb bleibt unklar, ob dieselben Schlüsse auch für die Privatversicherten gelten und wie sich die Versorgungssituation auf der Ebene der Gesamtbevölkerung entwickelt hat. Alles in allem erscheinen aber neue Finanzierungssysteme zwar weiterhin auf dem Vormarsch zu sein [22, 42, 43], jedoch oft bei ungenügender Evaluation ihrer versorgungsepidemiologischen Effekte.

\section{Ausblick: Revolution in der Schweiz?!}

Obwohl die Schweiz nicht gerade den Ruf eines Hortes revolutionärer Umtriebe besitzt, so war doch die Einführung heroingestützter Behandlungsformen in der Suchthilfe eine für Europa beispielgebende Umwälzung [44]. Bei der Finanzierung von stationärer Suchthilfe ist gegenwärtig ein zwar weniger spektakulärer, von den notwendigen Anstrengungen zur Überwindung zersplitterter Finanzierungswege her aber „revolutionärer“ Prozess im Gange: das Finanzierungskonzept FiSu/FiDé [45]. Ausgehend von einer gesamtschweizerischen Erhebung bei stationären Therapieeinrichtungen im Alkohol- und Drogenbereich für die Jahre 1995 bis 1998 [27], die detailliert die vielfach verzweigten Finanzierungswege der Institutionen in den verschiedenen Kantonen [46] erfasst hatte, wurde von der gleichnamigen FiSu-Kommission ein Konzept zur Vereinheitlichung der Therapiefinanzierung für die gesamte Schweiz erstellt [47]. Kernstück dieses Konzeptes ist es, die Zersplitterung von medizinischen, psychotherapeutischen und sozialarbeiterischen Leistungen in völlig disjunkte Erstattungsbereiche zu überwinden und künftig aus einer einzigen Quelle zu bezahlen in Form eines kantonalen Rechnungsfonds. In diesen zahlen die Wohnsitzkantone (die in der Schweiz für die Sozialhilfe zuständig sind), die KlientInnen (durch Abtretung ihrer allfälligen Rehabilitationsansprüche gegen die „Invalidenversicherung") sowie die Standortkantone der Therapieinstitutionen ein. Ausgeschüttet werden sollen die Mittel leistungsorientiert, wobei über die Schweiz hinweg einheitliche Tarife geplant und nach einer Pilotphase bereits kalkuliert sind [47]. Als erstattungsfähige Leistungen sollen für stationäre Therapien so genannte „Lebensfeld ersetzende Maßnahmen “7 in vier Sozialisationsbereichen de-

\footnotetext{
${ }^{7}$ Im Ersatz des (süchtigen) Lebensumfeldes liegt ja die eigentliche Indikation zur stationären Therapie.
}

finiert werden: 1. die Primärgruppe ersetzende Maßnahmen, 2. Schule und Bildung, 3. Arbeit sowie 4. altersspezifische Freizeitgestaltung. Pro Institution wird ein Profil erstellt, auf welchen Sozialisationsfeldern die betreffende Einrichtung Maßnahmen in welcher Dichte anbietet. Dabei wird nach Einzelleistungen, Leistungseinheit $(=$ „ein definiertes Paket gebündelter Einzelleistungen“) und Leistungsbereich (= „umfassende Planung und Realisierung des zu ersetzenden Sozialisationsfeldes“) unterschieden. Jede Institution kann dann pro Klient und Sozialisationsfeld die ihrem Angebotsprofil entsprechenden pauschalen Erlöse erzielen. Allein die Nomenklatur für die Leistungen scheint für traditionell medizinisch-psychiatrisch ausgebildete Ohren „gewöhnungsbedürftig“, bildet aber mit Sicherheit das Geschehen in stationären Suchteinrichtungen sehr viel klarer ab, als irgendwelche diagnoseorientierten Kostengruppen dies könnten. Nach Abschluss zweier Pilotversuche können sich einzelne Kantone ab 1.1.2003 entscheiden, ihr bisherigen Finanzierungssystem durch FiSu zu ersetzen oder dieses System zunächst beizubehalten und die „alten“ Bestimmungen der Invaliden-Versicherung zu reaktivieren (für ein ähnliches Prozedere im Alkoholbereich vgl. [48]). Die endgültige Einführung der FiSu-Systematik wird dann in komplizierten Gesprächen zwischen Bund und Kantonen geklärt. Eine Begleitforschung zur Evaluation der Auswirkungen auch hinsichtlich des Behandlungsergebnisses ist aber auf alle Fälle fest vorgesehen.

Es wird spannend sein, von den schweizerischen Ergebnissen zu erfahren. Mit der einheitlichen Finanzierung wäre auch die Grundlage gelegt, um zudem die Zersplitterung von somatischen, psychiatrischen und psychotherapeutischen Maßnahmen auf verschiedene Leistungsträger zu überwinden. Neuere Untersuchungen in den USA haben gezeigt, dass beispielweise AlkoholpatientInnen von einer integrierten Therapie bei einem Leistungserbringer einen besseren Erfolg in der Suchtbehandlung erwarten können [49], wenn sie zusätzlicher medizinisch-somatischer oder psychiatrischer Behandlung bedürfen.

\section{Literatur}

${ }^{1}$ Commander M, Odell S, Williams K et al. Pathways to care for alcohol use disorders. J Public Health Med 1999; 21 (1): 65-69

${ }^{2}$ Lundgren LM, Amodeo M, Ferguson F et al. Racial and ethnic differences in drug treatment entry of injection drug users in Massachusetts. J Substance Abuse Treatment 2001; 21: 145-153

${ }^{3}$ Walker L. Iatrogenic addiction and its treatment. Int J Addict 1978; 13 (3): $461-473$

${ }^{4}$ Werch C, Owen D. Iatrogrenic effects of alcohol and drug prevention programs. J Stud Alcohol 2002; 63 (5): 581 -590

${ }^{5}$ Wasem J, Hessel F, Kerim-Sade C. Methoden zur vergleichenden ökonomischen Evaluation von Therapien und zur rationalen Ressourcenallokation über Bereiche des Gesundheitswesens hinweg - Einführung, Vorteile, Risiken. Psychiatrische Praxis 2001; 28 (suppl. 1): $\mathrm{S} 12-\mathrm{S} 20$

${ }^{6}$ Mossialos E, Dixon A. Funding health care: an introduction. In: In: Mossialos E, Dixon A, Figueras JJK (Hrsg). Funding health care: options for Europe. Buckingham: Open University Press 2002; Chapter 1

${ }^{7}$ Systems EOoHC. Health Care Systems in Transition: Germany. Copenhagen: WHO Regional Office for Europe 2000

8 Systems EOoHC. Health Care Systems in Transition: Switzerland. Copenhagen: WHO Regional Office for Europe 2000

${ }_{9}$ Wagstaff A, van Doorslaer E, van der Burg H et al. Equity in the finance of health care: some further international comparisons. J Health Economics 1999; 11: $361-387$ 
${ }^{10}$ Shortt S. Medical savings accounts in publicly funded health care systems: enthusiasms versus evidence. CMAJ 2002; 167 (2): 159-162

${ }^{11}$ Murray C, Knaul F, Musgrove P et al. Defining and measuring fairness in financial contribution to the health system. Geneva: World Health Organization, without year; Report No.: GPE Discussion Paper Series: No. 24

12 Meyer PC, Denz MD. Sozialer Wandel der Arztrolle und der Ärzteschaft durch Manged Care in der Schweiz. Gesundheitswesen 2000; 62: $138-142$

${ }^{13}$ Chaix-Couturier C, Durand-Zaleski I, Jolly D et al. Effects of financial incentives on medical practice: results from a systematic review of the literature and methodological issues. Int J Qual Health Care 2000; $12(2): 133-142$

${ }^{14}$ Robinson J. Theory and practice in the design of physician payment incentives. Milbank Quarterly 2001; 79 (2): 149-177

${ }^{15}$ Gosden T, Forland F, Kristiansen IS et al. Impact of payment method on behaviour of primary care physicians: a systmatic review. J Health Serv Res Policy 2001; 6 (1): 44-55

${ }^{16}$ Armour BS, Pitts MM, Maclean R et al. The effect of explicit financial incentives on physician behavior. Arch Intern Med 2001; 161: $1261-1266$

17 Frick U, Binder H, Barta W et al. „Fair ist, Gleiches mit Gleichem zu vergleichen"? Eine Simulationsstudie zu den Krankenhausvergleichen nach $\S 5$ Bundespflegesatzverordnung. Gesundheitswesen 2003; 65, in press

${ }^{18}$ Frick U, Cording C, Rehm J et al. Herr Tur-Tur und die Krankenhausvergleiche: Ein Besuch in der Psychiatrie-Oase. Das Gesundheitswesen 2001; 63 (7): 435 - 439

${ }^{19}$ Jegers M, Kesteloot K, De Graeve D et al. A typology for provider payment systems in health care. Health Policy 2002; 60: 255-273

${ }^{20}$ Rauner MS, Schaffhauser-Linzatti MM. Impact of the new Austrian inpatient payment strategy on hospital behavior: a system-dynamics model. Socio-Economic Planning Sciences 2002; 36 (3): 161 - 182

${ }^{21}$ Dismuke CE, Sena V. Has DRG payment influenced the technical efficiency and productivity of diagnostic technologies in Portuguese public hospitals? An empirical analysis using parametric and nonparametric methods. Health Care Management Science 1999; 2: $107-116$

22 Beck K. Growing importance of capitation in Switzerland. Health Care Management Science 2000; 3: 111-119

${ }^{23}$ Mello MM, Stearns SC, Norton E. Do Medicare HMOs still reduce health services use after controlling for selection bias? Health Economics 2002; $11: 323-340$

${ }^{24}$ Frick U, Rehm J, John J et al. Substitutionspotentiale stationärer Leistungen im Land Salzburg: Eine mehrstufige Delphi-Befragung. Reihe Gesundheitsplanung. Salzburg: Amt der Landesregierung 1995

${ }^{25}$ Huber F, Hess K. HMO-Praxis - Ein alternatives Praxis-Krankenversicherungsmodell. In: Eichhorn S, Schmidt-Rettig B (Hrsg). Chancen und Risiken von Managed Care. Köln: Kohlhammer 1998; 245-259

${ }^{26}$ Schridde H, Blanke B. Finanzierungs- und Förderungsbedingungen von Suchtberatungsstellen in der Bundesrepublik Deutschland. Hannover. Centre for Health Economics and Health System Research 1998, May; Report No.: Diskussionspapier Nr. 16

${ }^{27}$ Herrmann H, Güntzel O, Simmel U et al. Stationäre Suchttherapie Schweiz: Angebot und Finanzierung. Bern: Bundesamt für Gesundheit 1999

${ }^{28}$ Zarkin G, Galinis D, French M et al. Financing strategies for drug abuse treatment programs. J Subst Abuse Treat 1995; 12 (6): 385-399

${ }^{29}$ Fischer W. Diagnosis Related Groups (DRGs) und Pflege - Grundlagen, Codierungssysteme, Integrationsmöglichkeiten. Bern: Huber 2002

${ }^{30}$ Michel E, Stiletto M, Gründler W et al. Diagnosencodierung und Erlös im AR-DRG-System. Gesundh ökon Qual manag 2002; 7 (5): 292 - 296
${ }^{31}$ Schenker L. Die Finanzierung der Schweizer Spitäler mit Hilfe von APDRG im Jahre 2002. Lausanne: Institut für Gesundheitsökonomie und -management der Universität Lausanne 2001

32 Fischer W. Diagnosis Related Groups (DRG's) und verwandte Patientenklassifikationssysteme. Wolfertswil (CH): Z/I/M 2000

${ }^{33}$ Rosenheck R, Massari L. Psychiatric inpatient care in the VA: before, during, and after DRG-based budgeting. Am J Psychiatry 1991; 148: $888-891$

34 BMAGS BfAGuS. Leistungsorientierte Krankenanstaltenfinanzierung LKF - Modell 2000. Wien: BMAGS 1999

${ }^{35}$ Frick U, Barta W, Zwisler R et al. Auswirkungen der Leistungsorientierten Krankenhausfinanzierung (LKF) auf die Verweildauern und Hospitalisierungen im Land Salzburg seit 1997. Gesundh ökon Qual manag 2001; 6: 95- 104

${ }^{36}$ Rauner MS, Schaffhauser-Linzatti MM. Evaluation of the new Austrian reimbursement system. In: De Angelis V, Ricciardi N, Strochi G (Hrsg). Monitoring, Evaluating, Planning Health Services - ORAHS '98. 24th Meeting of the European Working Group on Operational Research Applied to Health Sciences, July 19-24, 1998. Rome: World Scientific Publishing Co 1999; 221 - 233

${ }^{37}$ Sommersguter-Reichmann M. The impact of the Austrian hospital financing reform on hospital productivity: empirical evidence on efficiency and technology changes using a non-parametric input-based Malmquist approach. Health Care Management Science 2000; 3: 309-321

${ }^{38}$ Frick U, Barta W, Binder H. Fallpauschalen in der stationär-psychiatrischen Versorgung. Empirische Evaluation im Land Salzburg. Psychiat Prax 2001; 28 (suppl. 1): S55-S62

39 Bloom J, Hu T, Wallace N et al. Mental health costs and access under alternative capitation systems in Colorado. Health Services Research 2002; 37 (2): $315-340$

${ }^{40}$ Cuffel B, Bloom J, Wallace N et al. Two-Year outcome of fee-for-service and capitated Medicaid programs for people with severe mental illness. Health Services Research 2002; 37 (2): 341 - 359

${ }^{41}$ Magura S, Horgan C, Mertens J et al. Effects of managed care on alcohol and other drug (AOD) treatment. Alcohol Clin Exp Res 2002; 26 (3): $416-422$

42 Theurl E. Some aspects of the reform of the health care systems in Austria, Germany, and Switzerland. Health Care Analysis 1999; 7: $331-354$

${ }^{43}$ Riemer-Hommel P. The changing nature of contracts in German health care. Soc Sci Med 2002; 55 (8): 1447-1455

${ }^{44}$ Rehm J, Gschwend P, Steffen T et al. Feasibility, safety, and efficacy of injectable heroin prescription for refractory opioid addicts: a followup study. Lancet 2001; 358 (9291): 1417 - 1423

${ }^{45}$ Facheinheit SuA. Bericht zur Auswertung der Vernehmlassung über ein neues Finanzierungsmodell für Suchttherapien (FiSu 1999). Bern: Bundesamt für Gesundheit 1999

${ }^{46}$ Herrmann H, Simmel U. Kantonale Finanzierungsmechanismen im Bereich stationärer Drogentherapie. Basel/Bern: Schweizerische Koordinationsstelle für stationäre Therapieangebote im Drogenbereich 1999

${ }^{47}$ FiSu F. Pilotversuch und Trockenübung - Kurzfassungen. Bern: Kommission FiSu 2002

${ }^{48}$ Leslie D, Rosenheck R, White W. Capitated payments for mental health patients: a comparison of potential approaches in a public sector population. J Mental Health Policy Econ 2000; 3: 35-44

49 Weisner C, Mertens J, Parthasarathy S et al. Integrating primary medical care with addiction treatment. JAMA 2001; 286: 1715-1723 